\title{
Anisotropy Factors and Electromechanical Coupling in Lead-Free 1-3-Type Composites
}

\author{
Vitaly Yu. Topolov, Christopher R. Bowen, and Ashura N. Isaeva
}

\begin{abstract}
The effective electromechanical properties and anisotropy factors of novel lead-free 1-3-type composites are studied to demonstrate their large piezoelectric anisotropy and considerable level of electromechanical coupling. The composites studied contain two singlecrystal components and a polymer component. The first piezo-active component is a domain-engineered [001]poled single crystal based on ferroelectric alkali niobatestantalates, and this component is in the form of a system of long rods that are parallel to the poling axis $O X_{3}$. The second single-crystal component is a system of spheroidal piezoelectric $\mathrm{Li}_{2} \mathrm{~B}_{4} \mathrm{O}_{7}$ inclusions aligned in a continuous and relatively large polymer matrix. The single-crystal rods are surrounded by a single crystal / polymer matrix, and the connectivity of the composite is 1-0-3. It is shown that the conditions $d_{33}^{*} /\left|d_{31}^{*}\right| \geq 5$, which indicates a large degree of anisotropy of the piezoelectric coefficients, and $k_{33}^{*} /\left|k_{31}^{*}\right| \geq 5$ and $k_{t}^{*} /\left|k_{p}^{*}\right| \geq \mathbf{5}$, which indicate a large anisotropy of the electromechanical coupling factors, can be achieved simultaneously in specific ranges of the component volume fractions and inclusion aspect ratios. Moreover, in the same volume-fraction and aspect-ratio ranges, large electromechanical coupling factors $\left(k_{33}^{*} \approx k_{t}^{*} \approx 0.8-0.9\right)$ are also achieved. In this context, the important role of the elastic properties of the continuous anisotropic matrix is discussed. The properties and anisotropy factors of the lead-free 1-3-type composites are compared to similar parameters of conventional lead-containing piezoelectric materials, and the advantages of the composite system studied are described.
\end{abstract}

Index Terms - Ferroelectric Materials, Ferroelectric Properties, Piezoelectric and Ferroelectric Transducer Materials

This work was financially supported by the European Research Council under the European Union's Seventh Framework Programme (FP/2007-2013) / ERC Grant Agreement No. 320963 on Novel Energy Materials, Engineering Science and Integrated Systems (NEMESIS) and by the Southern Federal University ("Working out and creation of a hightechnological production of a mobile hydroacoustic complex to highlight a situation in various areas of the World ocean on the basis of modern piezoelectric means of the new generation", contract No. 03.G25.31.0276, May 29th, 2017).

V. Yu. Topolov is with the Department of Physics, Southern Federal University, 344090 Rostov-on-Don, Russia (e-mail: vutopolov@sfedu.ru).

C. R. Bowen is with the Department of Mechanical Engineering, University of Bath, Bath BA2 7AY, UK (e-mail: c.r.bowen@bath.ac.uk).

A. N. Isaeva is with IRC "Smart Materials", Southern Federal University, 344090 Rostov-on-Don, Russia (e-mail: aisaeva@sfedu.ru).

\section{INTRODUCTION}

The overwhelming majority of advanced piezo-active 1-3-type composites studied in the recent decades are based on relaxor-ferroelectric single crystals (SCs), for instance, $(1-x) \mathrm{Pb}\left(\mathrm{Mg}_{1 / 3} \mathrm{Nb}_{2 / 3}\right) \mathrm{O}_{3}-x \mathrm{PbTiO}_{3}(\mathrm{PMN}-x \mathrm{PT})$ or $(1$ $-x) \mathrm{Pb}\left(\mathrm{Zn}_{1 / 3} \mathrm{Nb}_{2 / 3}\right) \mathrm{O}_{3}-x \mathrm{PbTiO}_{3}$ [1] - [4]. These components with compositions near the morphotropic phase boundary are characterized by outstanding electromechanical properties that are known to strongly enhance the piezoelectric performance and related parameters of the composites. However, due to the lead-containing SC components, there is growing concern that their production may pollute the environment. As a result, there is increasing research effort to discover effective alternatives to lead-containing ferroelectric and piezoelectric materials [5] - [7], which also applies to the field of piezoelectric composites [8]. Among the promising highperformance piezoelectric materials, of particular interest are domain-engineered SCs based on alkali niobates-tantalates with piezoelectric coefficients $d_{3 j} \sim 10^{2} \mathrm{pC} / \mathrm{N}$ [9] - [11] that are comparable to the $d_{3 j}$ of conventional lead based $(\mathrm{Pb}$, $\mathrm{Zr}) \mathrm{TiO}_{3}$-type ferroelectric ceramics [4], [12]. Recently, examples of three-component lead-free 2-2-type [13] and 1-3type [14] composites with two single-crystal (SC) components and one polymer component and 1-3-type composites with one SC and two polymer components [15] have been reported that demonstrate their piezoelectric performance compared to lead-based materials and their application potential. To date, features of the piezoelectric anisotropy and related parameters of the lead-free SC-based composites have yet to be studied in detail. The aim of the present paper is to analyze anisotropy factors and electromechanical coupling of the novel lead-free 1-3-type composites with two SC components.

\section{Model And Effective Properties of the Composite}

It is assumed that the composite consists of a system of parallel SC rods surrounded by a SC / polymer matrix (Fig. 1). $\mathrm{SC}-1$ represents the domain-engineered ferroelectric SC component in the rod, and SC-2 represents the piezoelectric component represented as a system of spheroidal inclusions aligned in the polymer matrix, as indicated by the inset of Fig. 1. The SC-1 rods with square bases (Fig. 1) are characterized by a regular square arrangement in the $\left(\mathrm{X}_{1} O \mathrm{X}_{2}\right)$ plane. The crystallographic axes $X^{(1)}, Y^{(1)}$, and $Z^{(1)}$ of each SC-1 rod are oriented as follows: $X^{(1)}\left\|O X_{1}, Y^{(1)}\right\| O X_{2}$, and $Z^{(1)} \| O X_{3}$, and the spontaneous polarization of SC-1 is $\boldsymbol{P}_{\boldsymbol{s}}^{(\mathbf{1})} \uparrow \uparrow O X_{3}$. The shape of each SC-2 inclusion, as shown in the inset in Fig. 1, obeys the equation 


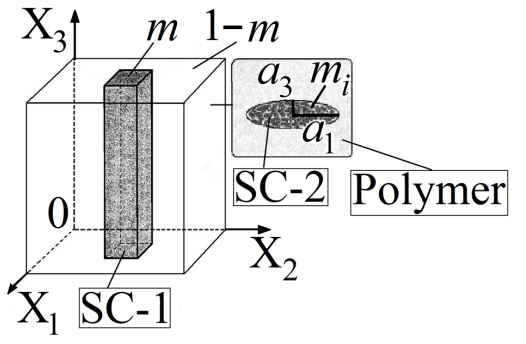

Fig. 1. Schematic of the $1-0-3 \mathrm{SC}-1 / \mathrm{SC}-2$ / polymer composite. $\left(X_{1} X_{2} X_{3}\right)$ is a rectangular coordinate system, $m$ and $1-m$ are volume fractions of SC-1 and surrounding SC-2/polymer matrix, respectively. In the inset, $m_{i}$ is the volume fraction of SC-2 in the polymer medium, and $a_{1}$ and $a_{3}$ are semi-axes of the SC-2 inclusion.

$$
\left(x_{1} / a_{1}\right)^{2}+\left(x_{2} / a_{1}\right)^{2}+\left(x_{3} / a_{3}\right)^{2}=1
$$

written in axes of the rectangular coordinate system $\left(X_{1} X_{2} X_{3}\right)$, and semi-axes of the spheroid from Eq. (1) are $a_{1}, a_{2}=a_{1}$ and $a_{3}$. Hereby the SC-2 / polymer matrix is characterized by $0-3$ connectivity. The SC-2 inclusions are regularly distributed in the polymer medium and occupy the sites of a simple tetragonal lattice with unit-cell vectors parallel to the $O X_{k}$ axes. By analogy with the $\mathrm{SC}-1$ rod, the orientation of the crystallographic axes $X^{(2)}, Y^{(2)}$, and $Z^{(2)}$ of each SC-2 inclusion obeys the conditions $X^{(2)}\left\|O X_{1}, Y^{(2)}\right\| O X_{2}$, and $Z^{(2)} \| O X_{3}$. The shape of the SC-2 inclusion is described by the aspect ratio $\rho_{i}=$ $a_{1} / a_{3}$ that is fixed over the composite sample. The radius or the largest semi-axis of the SC-2 inclusion is assumed to be much lower than the length of the side of the square that is the intersection of the $\mathrm{SC}$ rod with the $\left(X_{1} O X_{2}\right)$ plane (Fig. 1). This assumption enables us to apply the long-wave approximation to determine the effective properties of the composite. It should be added that the presence of one ferroelectric component (SC-1) and the orientation of the SC-1 rods parallel to the poling axis $O X_{3}$, as in Fig. 1, facilitates poling of the composite sample as a whole.

The composite shown in Fig. 1 is characterized by $1-0-3$ connectivity, and the effective electromechanical properties of this composite are evaluated in two stages [4], [16]. In the first stage, the effective properties of the matrix (i.e., $0-3 \mathrm{SC}-2 /$ polymer composite) are determined by means of the effective field method [4] that takes into consideration an electromechanical interaction between the piezoelectric inclusions. In the effective field method, this interaction is described in terms of a local field that acts on each inclusion in the 0-3 composite, and this field is determined by taking into account boundary conditions at the inclusion - matrix interface. Effective properties of the 0-3 composite are described by the $9 \times 9$ matrix $\left\|C^{(0-3)}\right\|=\left\|C^{(p)}\right\|+m_{i}\left(\left\|C^{(S C)}\right\|-\right.$ $\left.\left\|C^{(p)}\right\|\right)\left[\|I\|+\left(1-m_{i}\right)\|S\|\left\|C^{(p)}\right\|^{-1}\left(\left\|C^{(S C)}\right\|-\left\|C^{(p)}\right\|\right)\right]^{-1}$, where $\left\|C^{(S C)}\right\|$ and $\left\|C^{(p)}\right\|$ are matrices of electromechanical constants of the SC-2 and polymer, respectively, $m_{i}$ is the volume fraction of SC-2, $\|I\|$ is the identity matrix, and $\|S\|$ is the matrix that contains the Eshelby tensor components depending on $\rho_{i}$ and elements of $\left\|C^{(2)}\right\|$.

In the second stage, we apply the matrix method [4] that allows for the electromechanical interaction between the SC-1 rods and 0-3 matrix (Fig. 1), and the properties of the 0-3 matrix are given by $\left\|C^{(0-3)}\right\|$. Periodic boundary conditions are enforced on interfaces $x_{a}=$ const ( $a=1$ and 2). For instance, at $x_{1}=$ const, components of mechanical stress $\sigma_{11}, \sigma_{12}$ and $\sigma_{13}$, strain $\xi_{22}, \xi_{23}$ and $\xi_{33}$, electric displacement $D_{1}$, and electric field $E_{2}$ and $E_{3}$ are to be continuous. The effective properties of the 1-0-3 composite are found from the matrix $\left\|C^{*}\right\|=$ $\left[\left\|C^{(1)}\right\| \cdot\|M\| m+\left\|C^{(0-3)}\right\|(1-m)\right] \cdot[\|M\| m+\|I\|(1-m)]^{-1}$, where $\left\|C^{(1)}\right\|$ characterizes the properties of SC-1, and $\|M\|$ is concerned with the aforementioned periodic boundary conditions [4], [16] and expressed in terms of elements of $\left\|C^{(1)}\right\|$ and $\left\|C^{(0-3)}\right\|$. The $\left\|C^{*}\right\|$ matrix is written in the form

$$
\left\|C^{*}\left(m, \rho_{i}, m_{i}\right)\right\|=\left(\begin{array}{l}
\left\|s^{* E}\right\|\left\|d^{*}\right\|^{t} \\
\left\|d^{*}\right\|\left\|\varepsilon^{* \sigma}\right\|
\end{array}\right) .
$$

In Eq. (2), $\left\|s^{*}\right\|$ is the $6 \times 6$ matrix of elastic compliances at $E=$ const, $\left\|d^{*}\right\|$ is the $3 \times 6$ matrix of piezoelectric coefficients, $\left\|\varepsilon^{* \sigma}\right\|$ is the $3 \times 3$ matrix of dielectric permittivities at $\sigma=$ const, and ' $t$ ' denotes transposition. It should be noted that the effective field and matrix methods are used to predict the effective electromechanical properties of many piezo-active composites. Examples of application of these methods and comparison of results obtained using different methods are highlighted in work [4]. Based on elements of $\left\|C^{*}\right\|$ from Eq. (2), one can find the piezoelectric coefficients $g_{i j}^{*}$ of the composite using the relation [17]

$$
\left\|g^{*}\right\|=\left\|\varepsilon^{* \sigma}\right\|^{-1} \cdot\left\|d^{*}\right\| \text {. }
$$

In the present paper, we analyze the electromechanical coupling factors (ECFs) $k_{3 l}^{*}=d_{3 l}^{*} /\left(\varepsilon_{33}^{*} s_{l l}^{*}\right)^{1 / 2}$ (longitudinal oscillation mode at $l=3$ and transverse oscillation modes at $l=$ 1 or $l=2), k_{t}^{*}=e_{33}^{*} /\left(c_{33}^{*} \varepsilon_{33}^{*}\right)^{1 / 2}$ (thickness oscillation mode), $k_{p}^{*}=$ $k_{31}^{*}\left[2 s_{11}^{*} E /\left(s_{11}^{*}+s_{12}^{*} E\right)\right]^{1 / 2}$ (planar oscillation mode), and the anisotropy factors

$$
\zeta_{1}^{*}=d_{33}^{*} / d_{31}^{*}, \zeta_{2}^{*}=k_{33}^{*} / k_{31}^{*}=\zeta_{1}^{*}\left(s_{11}^{*} E / s_{33}^{* E}\right)^{1.2} \text {, and } \zeta_{3}^{*}=k_{t}^{*} / k_{p}^{*},
$$

where $c_{33}^{* D}$ is the longitudinal elastic modulus at $D=$ const, and $\varepsilon_{33}^{* \xi}$ is the dielectric permittivity at $\xi=$ const. The anisotropy factors from Eqs. (4) are concerned with either the piezoelectric activity $\left(\zeta_{1}^{*}\right)$ or ECFs $\left(\zeta_{2}^{*}\right.$ and $\left.\zeta_{3}^{*}\right)$ of the composite. In the present study, we do not analyze the anisotropy factor $\zeta_{e}^{*}=e_{33}^{*} / e_{31}^{*}$ that is related to the piezoelectric coefficients $e_{i j}^{*}$, since the condition $\left|\zeta_{e}^{*}\right|>>1$ holds [4] for various 1-3-type composites due their columnar structure, i.e., a system of piezoelectric rods (Fig. 1) oriented parallel to the poling axis $O X_{3}$.

\section{ANISOTROPY FACTORS AND ELECTROMECHANICAL COUPLING}

Among the lead-free components to be considered in this work, we consider the [001]-poled domain-engineered ferroelectric KNNTL and KNN-T SCs (Table I, SC-1 components), piezoelectric $\mathrm{Li}_{2} \mathrm{~B}_{4} \mathrm{O}_{7} \quad$ (LBO) $\quad \mathrm{SC} \quad$ (SC-2 component), and piezo-passive polymers, namely, polyethylene (PE) and polyurethane (Table II). The SC-1 and SC-2 components of the studied composites are described by $4 \mathrm{~mm}$ symmetry. The LBO SC is a unique piezoelectric component since the signs of its piezoelectric coefficients $e_{i j}$ (see Table II) 
TABLE I

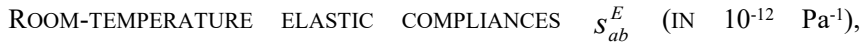
PIEZOELECTRIC COEFFICIENTS $d_{i j}$ (IN PC / N), AND DIELECTRIC PERMITTIVITY $\varepsilon_{p p}^{\sigma}$ OF [001]-POLED DOMAIN-ENGINEERED SCS $(4 \mathrm{~mm}$ SYMMETRY)

\begin{tabular}{lcc}
\hline \hline $\begin{array}{c}\text { Electromechanical } \\
\text { constants }\end{array}$ & $\mathrm{KNNTL}^{\mathrm{a}}[11]$ & $\mathrm{KNN}^{\mathrm{b}}[10]$ \\
\hline$s_{11}^{E}$ & 33.4 & 11.9 \\
$s_{12}^{E}$ & -7.36 & -4.30 \\
$s_{13}^{E}$ & -25.8 & -5.60 \\
$s_{33}^{E}$ & 57.7 & 15.5 \\
$s_{44}^{E}$ & 12.8 & 12.0 \\
$s_{66}^{E}$ & 13.5 & 10.7 \\
$d_{15}$ & 66 & 45.0 \\
$d_{31}$ & -260 & -77 \\
$d_{33} / \varepsilon_{0}$ & 545 & 162 \\
$\varepsilon_{11}^{\sigma} \varepsilon_{0}$ & 400 & 291 \\
$\varepsilon_{33}^{\sigma} \varepsilon_{0}$ & 650 & 267 \\
\end{tabular}

${ }^{\mathrm{a}}\left[\mathrm{Li}_{x}\left(\mathrm{~K}_{1-y} \mathrm{Na}_{y}\right)_{1-x}\right]\left(\mathrm{Nb}_{1-z} \mathrm{Ta}_{z}\right) \mathrm{O}_{3}: \mathrm{Mn}$, where $x=0.06, y=0.1-0.3, z=0.07-$ 0.17 , and the level of Mn doping is $0.25 \mathrm{~mol} . \%$, Ref. 11

${ }^{\mathrm{b}}\left(\mathrm{K}_{0,562} \mathrm{Na}_{0,438}\right)\left(\mathrm{Nb}_{0,768} \mathrm{Ta}_{0,232}\right) \mathrm{O}_{3}$, Ref. 10

\section{TABLE II}

ROOM-TEMPERATURE ELASTIC MODULi $c_{a b}^{E}$ (IN $10^{10} \mathrm{~Pa}$ ), PIEZOELECTRIC COEFFICIENTS $e_{i j}\left(\mathrm{IN} \mathrm{C} / \mathrm{m}^{2}\right.$ ), AND DIELECTRIC PERMITTIVITY $\varepsilon_{p p}^{\xi}$ OF SC-2 AND POLYMER COMPONENTS

\begin{tabular}{lccc}
\hline \hline $\begin{array}{c}\text { Electromechanical } \\
\text { constants }\end{array}$ & $\begin{array}{c}\text { LBO SC } \\
{[18]}\end{array}$ & $\begin{array}{c}\text { PE } \\
{[19,20]}\end{array}$ & $\begin{array}{c}\text { Polyurethane } \\
{[21]}\end{array}$ \\
\hline$c_{11}^{E}$ & 13.5 & 0.0778 & 0.442 \\
$c_{12}^{E}$ & 0.357 & 0.0195 & 0.260 \\
$c_{13}^{E}$ & 3.35 & 0.0195 & 0.260 \\
$c_{33}^{E}$ & 5.68 & 0.0778 & 0.442 \\
$c_{44}^{E}$ & 5.85 & 0.0292 & 0.091 \\
$c_{66}^{E}$ & 4.67 & 0.0292 & 0.091 \\
$e_{15}$ & 0.472 & 0 & 0 \\
$e_{31}$ & 0.290 & 0 & 0 \\
$e_{33}$ & 0.928 & 0 & 0 \\
$\varepsilon_{11}^{\xi} / \varepsilon_{0}$ & 8.90 & 2.3 & 3.5 \\
$\varepsilon_{33}^{\xi} / \varepsilon_{0}$ & 8.07 & 2.3 & 3.5 \\
--------------------------------------------------------------------------------
\end{tabular}

coincide with the signs of $e_{i j}$ of the highly anisotropic $\mathrm{PbTiO}_{3}-$ type ceramics $[4,12]$. However the piezoelectric effect in the $\mathrm{LBO} \mathrm{SC}$ is weaker than that in the poled $\mathrm{PbTiO}_{3}$-type ceramics. An unusual feature of the LBO SC is that it exhibits a considerable degree of elastic anisotropy, with large ratios of its elastic moduli $c_{13}^{E} / c_{12}^{E} \approx 9.4$ and $c_{11}^{E} / c_{12}^{E} \approx 38$ (see data in Table II). These large ratios have no analogies with other ferroelectric ceramics and domain-engineered ferroelectric SCs; see, for instance, experimental data in work [3], [4], [9] - [12].

In Section III we consider examples of the large anisotropy factors from Eqs. (4). We show that conditions

$$
\begin{aligned}
& \left|\zeta_{k}^{*}\right| \geq 5, \\
& \zeta_{2} \mid \geq 5,
\end{aligned}
$$

and

$$
\left|\zeta_{3}^{*}\right| \geq 5
$$

hold in specific volume-fraction $\left(m\right.$ and $\left.m_{i}\right)$ and aspect-ratio $\left(\rho_{i}\right)$ ranges. The large $\left|\zeta_{j}^{*}\right|$ values are of interest to a transducer designer as it provides an opportunity to exploit the composite in a device that uses the longitudinal (or thickness) oscillation mode [4], [22] - [25] while suppressing the transverse (or planar) oscillation mode to a large extent due to the restricted piezoelectric response related to $d_{31}^{*}, k_{31}^{*}$, or $k_{p}^{*}$ from Eqs. (4).

Such transducers are employed in structural health monitoring (where the longitudinal mode is exploited) [23] or in ultrasonic devices (where the thickness mode is exploited) [24, 25].

The anisotropy factors from conditions (5) - (7) are restricted by the factor 5 . This stems from the condition $\left|d_{33} / d_{31}\right| \approx 5$ that is related to single-domain $\mathrm{PbTiO}_{3} \mathrm{SCs}$ [26], [27] at room temperature, in the ferroelectric $4 \mathrm{~mm}$ phase. In comparison to the $\mathrm{PbTiO}_{3} \mathrm{SC}$, poled modified $\mathrm{PbTiO}_{3}$ ceramics are characterized [27] by the larger anisotropy of the piezoelectric coefficients $d_{3 j}$, ECFs $\left|k_{t} / k_{p}\right|$, etc.

\section{A. Polyethylene-Containing Composites,}

The first example of composite performance is illustrated by Fig. 2 and is related to composites with highly oblate LBO SC inclusions in a $0-3$ matrix, see the inset in Fig. 1. Figure 2 suggests that the simultaneous validity of conditions (5)-(7) is achieved for a range of conditions, apart for curves 3 and 6 in Fig 2(c). A simultaneous decrease of $\left|\zeta_{j}^{*}\right|$ at a volume fraction of SC- $1 m>0.2$ is a result of the strong influence of the domain-engineered SC-1 rods on piezoelectric activity [14] and the ECFs of the composite. As is known from Table I, both the domain-engineered SCs are characterized by a moderate anisotropy of the piezoelectric coefficients $d_{3 j}$. At a volume fraction $m<0.2$, the influence of the elastic properties of the 0-3 matrix is stronger, irrespective of the main piezoelectric component, i.e., SC-1 in rods. The configuration and arrangement of $\min \zeta_{j}^{*}$ in Fig. 2 enable us to assume that the elastic properties of the 0-3 matrix influence the anisotropy factors $\zeta_{j}^{*}$ in Eqs. (4), however the level of influence varies for different anisotropy factors $\zeta_{j}^{*}$. This can be also related with the specific boundary conditions for the SC-1 rods in the 1-3-type composite structure of Fig. 1, with the presence of the $0-3$ matrix exhibiting a weak piezoelectric response, and with the heavily oblate SC-2 inclusions in the matrix. In addition, the graphs in Fig. 2(b) and (d) show that the influence of the elastic properties of the $0-3$ matrix on $\zeta_{j}^{*}$ is appreciable even at small volume fractions of SC-2 $m_{i}=$ 0.05 ; see curves $1-3$.

The second example is illustrated by Table III where we show the volume-fraction $(m)$ ranges of the validity of conditions 

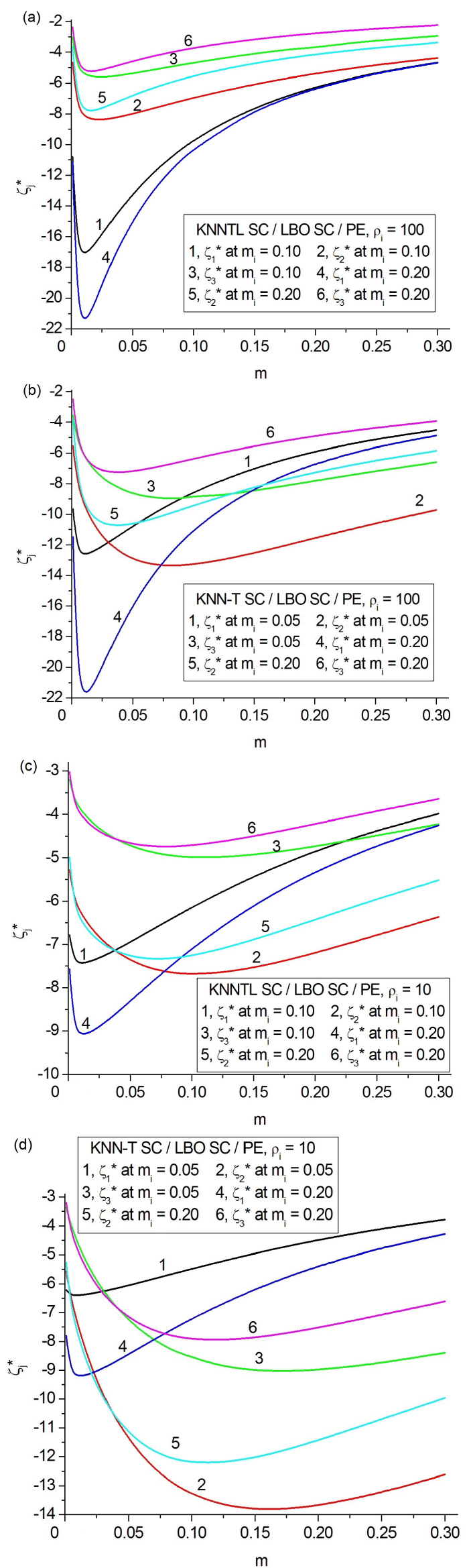

Fig. 2. Anisotropy factors $\zeta_{j}^{*}$ of 1-0-3 composites at $\rho_{i}=100$ (a and (b), and at $\rho_{i}=10$ (c and d).
TABLE III

VOLUME-FRACTION $(m)$ RANGES WHEREIN CONDITIONS (5), (6), AND (7) HOLD IN $1-0-3$ COMPOSITES AT $m_{i}=0.1$ AND $0.01 \leq \rho_{i} \leq 100$

\begin{tabular}{|c|c|c|c|}
\hline$\rho_{i}$ & {$\left[m_{1 a} ; m_{1 b}\right]$} & {$\left[m_{2 a} ; m_{2 b}\right]$} & {$\left[m_{3 a} ; m_{3 b}\right]$} \\
\hline \multicolumn{4}{|c|}{ KNN-T SC / LBO SC / PE composite } \\
\hline 0.01 & {$[0.001 ; 0.032]$} & {$[0.001 ; 0.871]$} & {$[0.001 ; 0.725]$} \\
\hline 0.1 & {$[0.001 ; 0.017]$} & {$[0.001 ; 0.870]$} & {$[0.001 ; 0.722]$} \\
\hline 0.5 & {$[0.001 ; 0.017]$} & {$[0.001 ; 0.867]$} & {$[0.022 ; 0.717]$} \\
\hline 1 & {$[0.001 ; 0.040]$} & {$[0.001 ; 0.864]$} & {$[0.023 ; 0.708]$} \\
\hline 2 & {$[0.001 ; 0.077]$} & {$[0.001 ; 0.856]$} & {$[0.022 ; 0.695]$} \\
\hline 5 & {$[0.001 ; 0.139]$} & {$[0.001 ; 0.836]$} & {$[0.017 ; 0.661]$} \\
\hline 10 & {$[0.001 ; 0.190]$} & {$[0.001 ; 0.806]$} & {$[0.012 ; 0.614]$} \\
\hline 20 & {$[0.001 ; 0.234]$} & {$[0.001 ; 0.756]$} & {$[0.008 ; 0.542]$} \\
\hline 50 & {$[0.001 ; 0.271]$} & {$[0.001 ; 0.653]$} & {$[0.004 ; 0.422]$} \\
\hline 100 & {$[0.001 ; 0.281]$} & {$[0.001 ; 0.558]$} & {$[0.004 ; 0.330]$} \\
\hline \multicolumn{4}{|c|}{ KNNTL SC / LBO SC / PE composite } \\
\hline 0.01 & {$[0.001 ; 0.026]$} & {$[0.001 ; 0.617]$} & {$[0.001 ; 0.343]$} \\
\hline 0.1 & {$[0.001 ; 0.013]$} & {$[0.001 ; 0.591]$} & {$[0.027 ; 0.241]$} \\
\hline 0.5 & {$[0.001 ; 0.010]$} & {$[0.001 ; 0.574]$} & $--^{a}$ \\
\hline 1 & {$[0.001 ; 0.033]$} & {$[0.001 ; 0.566]$} & $-{ }^{\mathrm{a}}$ \\
\hline 2 & {$[0.001 ; 0.072]$} & {$[0.001 ; 0.552]$} & $-^{\mathrm{a}}$ \\
\hline 5 & {$[0.001 ; 0.135]$} & {$[0.001 ; 0.519]$} & $-^{\mathrm{a}}$ \\
\hline 10 & {$[0.001 ; 0.186]$} & {$[0.001 ; 0.474]$} & $-^{\mathrm{a}}$ \\
\hline 20 & {$[0.001 ; 0.230]$} & {$[0.001 ; 0.411]$} & {$[0.039 ; 0.136]$} \\
\hline 50 & {$[0.001 ; 0.266]$} & {$[0.001 ; 0.310]$} & {$[0.009 ; 0.108]$} \\
\hline 100 & {$[0.001 ; 0.273]$} & {$[0.001 ; 0.234]$} & {$[0.006 ; 0.077]$} \\
\hline
\end{tabular}

${ }^{a}$ Condition (7) is violated

volume fraction of SC-2 at $m_{i}=0.1$ means that the spheroidal inclusions influence the elastic properties of the $0-3$ matrix to a restricted degree. The volume-fraction $(m)$ range, wherein condition (5) holds for the composite, is represented as [ $m_{1 a}$; $\left.m_{1 b}\right]$. By analogy with this notation, we introduce the volumefraction ranges $\left[m_{2 a} ; m_{2 b}\right]$ and $\left[m_{3} a ; m_{3 b}\right]$ wherein conditions (6) and (7) hold, respectively. As follows from Table III, the volume-fraction range $\left[m_{3 a} ; m_{3 b}\right]$ cannot be found for the KNNTL-based composite at $0.1<\rho_{i}<20$. This can be due to the relatively small difference between the elastic properties of the KNNTL SC rod and the 0-3 matrix that surrounds the rod.

In the third example, we show the simultaneous validity of conditions (5)-(7) and large values of the ECFs $k_{33}^{*}$ and $k_{t}^{*}$ in a

wide range of aspect-ratios $\left(\rho_{i}\right)$, see Fig. 3 . We consider the case of the volume fraction of SC- $1 m=0.15$, and at this volume fraction, irrespective of $\rho_{i}$, we see that the piezoelectric coefficient $d_{33}^{*}$ and ECF $k_{33}^{*}$ obey the conditions $0.9 d_{33}^{(1)}<d_{33}^{*}<d_{33}^{(1)}$ and $0.9 k_{33}^{(1)}<k_{33}^{*}<k_{33}^{(1)}$, where superscript '(1)' denotes the SC-1 properties. The graphs in Fig. 3 suggest that the equality $k_{33}^{*} \approx k_{t}^{*}$ is valid in a wide $\rho_{i}$ range, and such a performance is typical of 1-3-type composites [4], [25].

\section{B. Influence of Matrix Composition}

The next example is related to the piezoelectric anisotropy of polyurethane-containing composites. We now replace PE with polyurethane in the 0-3 matrix which leads to a smaller difference between the elastic properties of the SC-2 and polymer components in the $0-3$ matrix. As a consequence, no simultaneous validity of conditions (5)-(7) is observed for the studied composites. However, one can observe restricted volume-fraction ( $m$ and $m_{i}$ ) ranges wherein condition (5) holds for the KNN-T-based composite, see Fig. 4. The small volume fractions of SC-1 $(0.02<m<0.05)$, which are related to $\min \zeta_{1}^{*}$ in Fig. 4, cannot lead to active applications of the KNN-T SC/ 

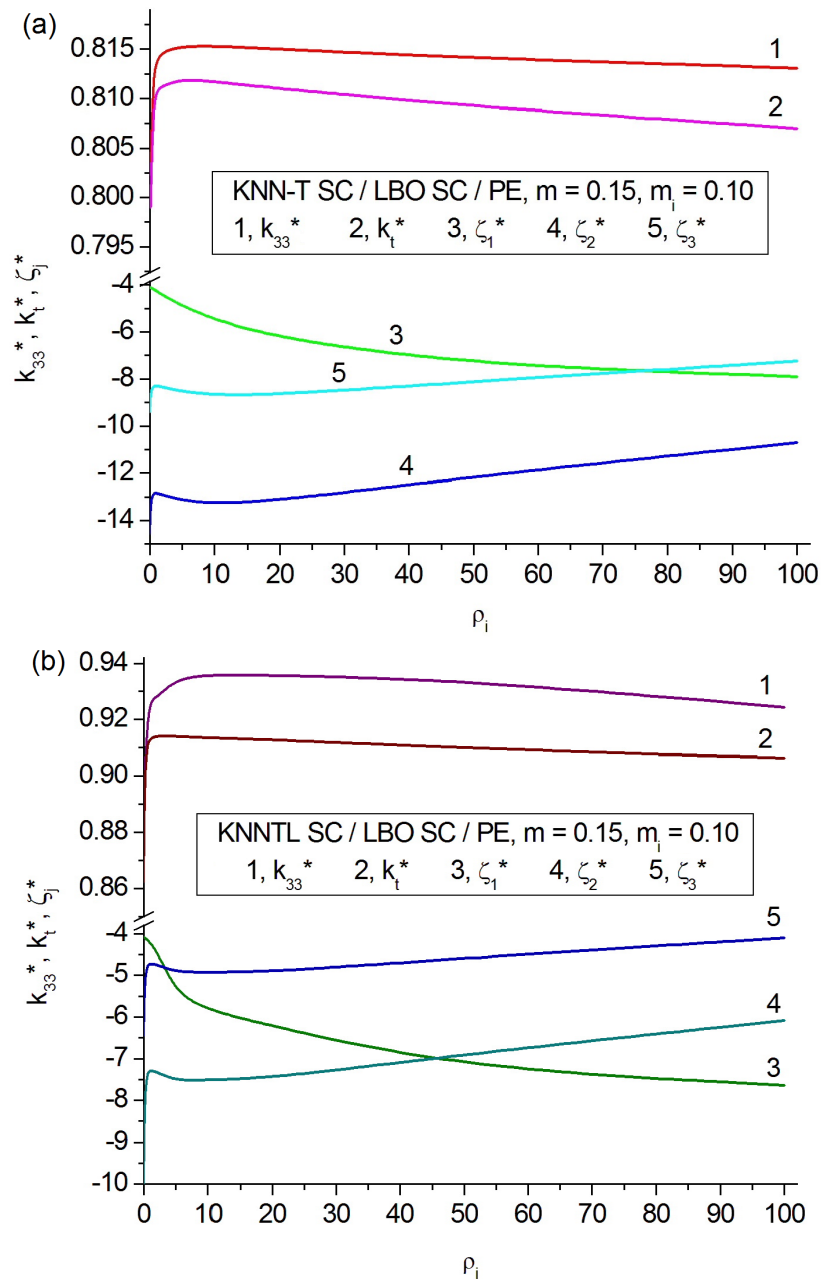

Fig. 3. ECFs $k_{33}^{*}$ and $k_{t}^{*}$ and anisotropy factors $\zeta_{j}^{*}$ of 1-0-3 composites at $m=0.15$ and $m_{i}=0.10$.

LBO SC / polyurethane composite as a material with a large anisotropy of $d_{3 j}^{*}$ because of the relatively small $\left|d_{3 j}^{*}\right|$ values.

For instance, at $m=0.02$ and $\rho_{i}=100$, for the $1-0-3 \mathrm{KNN}-\mathrm{T}$ SC / LBO SC / polyurethane composite, we have $d_{33}^{*}=41.7$ $\mathrm{pC} / \mathrm{N}$ and $d_{31}^{*}=-8.55 \mathrm{pC} / \mathrm{N}\left(m_{i}=0.10\right), d_{33}^{*}=38.7 \mathrm{pC} / \mathrm{N}$ and $d_{31}^{*}=-7.01 \mathrm{pC} / \mathrm{N}\left(m_{i}=0.15\right), d_{33}^{*}=34.6 \mathrm{pC} / \mathrm{N}$ and $d_{31}^{*}=-5.45$ $\mathrm{pC} / \mathrm{N}\left(m_{i}=0.25\right)$, etc. These $\left|d_{3 j}^{*}\right|$ values are smaller than the $\left|d_{3 j}\right|$ values of highly anisotropic modified $\mathrm{PbTiO}_{3}$ ceramics [12].

\section{Features of Elastic Properties of the 0-3Matrix}

As follows from Eqs. (4), the elastic properties of the composite influence the ECFs and anisotropy factors, in particular $\zeta_{2}^{*}$ and $\zeta_{3}^{*}$. The data in Table I suggest that both domain-engineered SCs exhibit a similar moderate anisotropy of elastic and piezoelectric properties. An important potential to influence the anisotropy factors $\zeta_{j}^{*}$ from Eqs. (4) can be 'hidden' in the 0-3 matrix. This stems from the fact that the 0-3 matrix exhibits weak piezoelectric and dielectric properties (i.e., $\left|d_{3 j}^{(0-3)}\right|<<\left|d_{3 j}^{*}\right|$ and $\varepsilon_{p p}^{(0-3), \sigma<<} \varepsilon_{33}^{* \sigma}$ ) and a considerable elastic anisotropy in specific $m_{i}$ and $\rho_{i}$ ranges.

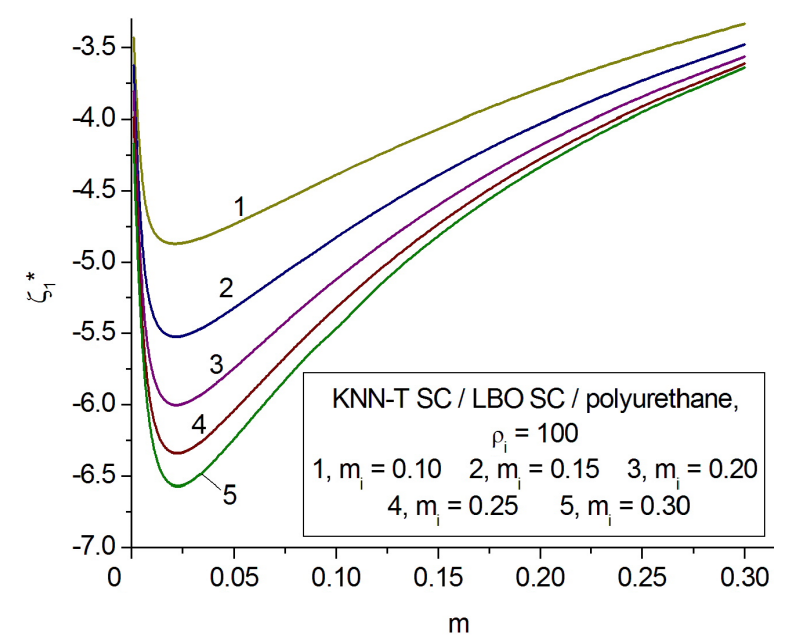

Fig. 4. Anisotropy factor $\zeta_{1}^{*}$ of the $1-0-3 \mathrm{KNN}-\mathrm{T} \mathrm{SC} / \mathrm{LBO} \mathrm{SC} /$ polyurethane composite at $\rho_{i}=100$.

(a)
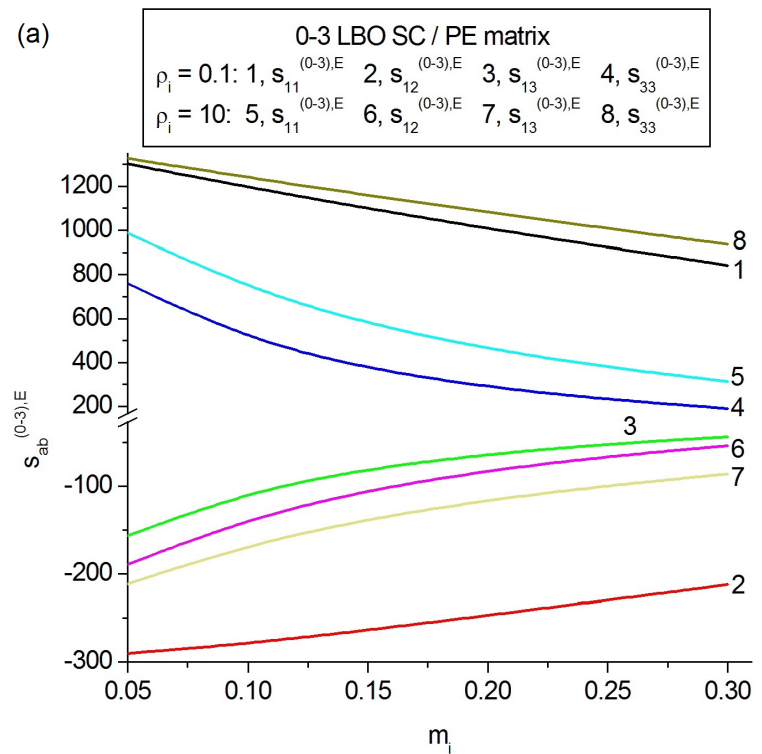

(b)

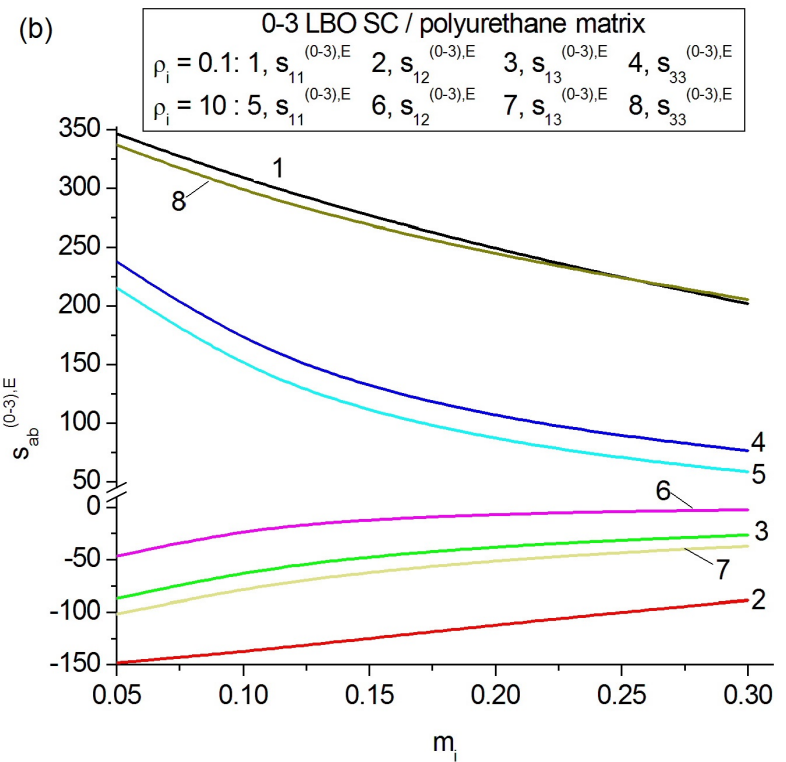

Fig. 5. Elastic compliances $s_{t a b}^{(0-3), E}$ of the 0-3 LBO SC / polymer matrix with either prolate $\left(\rho_{i}=0.1\right)$ or oblate $\left(\rho_{i}=10\right)$ inclusions. 
Examples of the volume-fraction $\left(m_{i}\right)$ dependence of the elastic compliances $s_{a b}^{(0-3), E}$ of the 0-3 SC-2 / polymer matrix are shown in Fig. 5. We consider $s_{a b}^{(0-3), E}$ for $a b=11,12,13$, and 33, while these elastic compliances influence the elastic compliances $s_{a b}^{*}$, piezoelectric coefficients $d_{3 j}^{*}$, and anisotropy factors $\zeta_{j}^{*}$ of the composite to a certain degree. In Fig. 5(a) we show the considerable elastic anisotropy related with the following ratios in the PE-containing 0-3 matrix: $s_{11}^{(0-3), E} / s_{13}^{(0-3), E}, s_{33}^{(0-3), E} / s_{13}^{(0-3), E}$, and $s_{11}^{(0-3), E} / s_{33}^{(0-3), E}$. On increasing $m_{i}$, the elastic anisotropy of the $0-3$ matrix weakens.

In comparison to the data in Fig. 5(a), absolute values of $s_{a b}^{(0-3), E}$ in Fig. 5(b) become smaller, and the elastic anisotropy of the 0-3 matrix becomes less appreciable. This is due to the presence of polyurethane instead of PE and the larger elastic stiffness of polyurethane in comparison to $\mathrm{PE}$, see data in Table II. As a result, the 0-3 LBO SC / polyurethane matrix leads to a smaller anisotropy of the piezoelectric properties and ECFs of the 1-0-3 polyurethane-containing composite, especially at volume fractions of SC-1 $m<0.2$. This is consistent with our results represented in Section III.B.

\section{Electromechanical Coupling Factor $k_{t}^{*}$}

The large ECF $k_{t}^{*}$ and the validity of condition (7) are of interest in transducer applications operating with a thickness oscillation mode. Data on the volume-fraction range $\left[m_{3} ; m_{3 b}\right]$ in the 4th column of Table III and the graphs in Fig. 6 show that large $k_{t}^{*}$ values are often achieved with large $\left|\zeta_{3}^{*}\right|$ values. The 1-3-type composite structure leads to a large increase of $k_{t}^{*}$ for relatively small volume fractions of SC-1 $m$ and to a diffuse local $\max k_{t}^{*}$ at $m>0.5$. In the latter case, the influence of the SC-2 component and aspect ratio of the SC-2 inclusions $\rho_{i}$ on $k_{t}^{*}$ is moderate, and we observe only minor changes in the volume-fraction $(m)$ dependence of $k_{t}^{*}$ at variations of $\rho_{i}$, see Fig. 6. In this regard, changes in the microgeometry of the 0-3 matrix with a weak piezoelectric activity have only a restricted influence of the electromechanical coupling along the poling axis $O X_{3}$. It should be added that values of local $\max _{k}^{*}$ of the KNNTL-based composite, Fig. 6(c), are approximately 1.7-1.8 times larger than $k_{t}^{(1)}$ of the KNNTL SC [11], and such an increase of $k_{t}^{*}$ is more pronounced than that in 1-3 ceramic / polymer composites [25]. In our opinion, the reason for the large ECF $k_{t}^{*}$ in the studied volume-fraction and aspect-ratio ranges is mainly a result of the successful combination of elastic properties of the SC-1 and 0-3 matrix in the 1-3-type composite. We also observe a relatively small difference between the values of local $\max k_{t}^{*}$ of the KNN-Tbased and KNNTL-based composites [see, e.g., Fig. 6(b) and (c)], and this is observed despite the clear difference between the properties of the SC-1 components listed in Table I. The results shown in Fig. 6 and the volume-faction ranges $\left[m_{3 a}\right.$; $m_{3 b}$ ], wherein a large anisotropy of ECFs is achieved, suggest that the studied 1-3-type composites can be of interest as piezoelectric transducers that use the thickness mode.
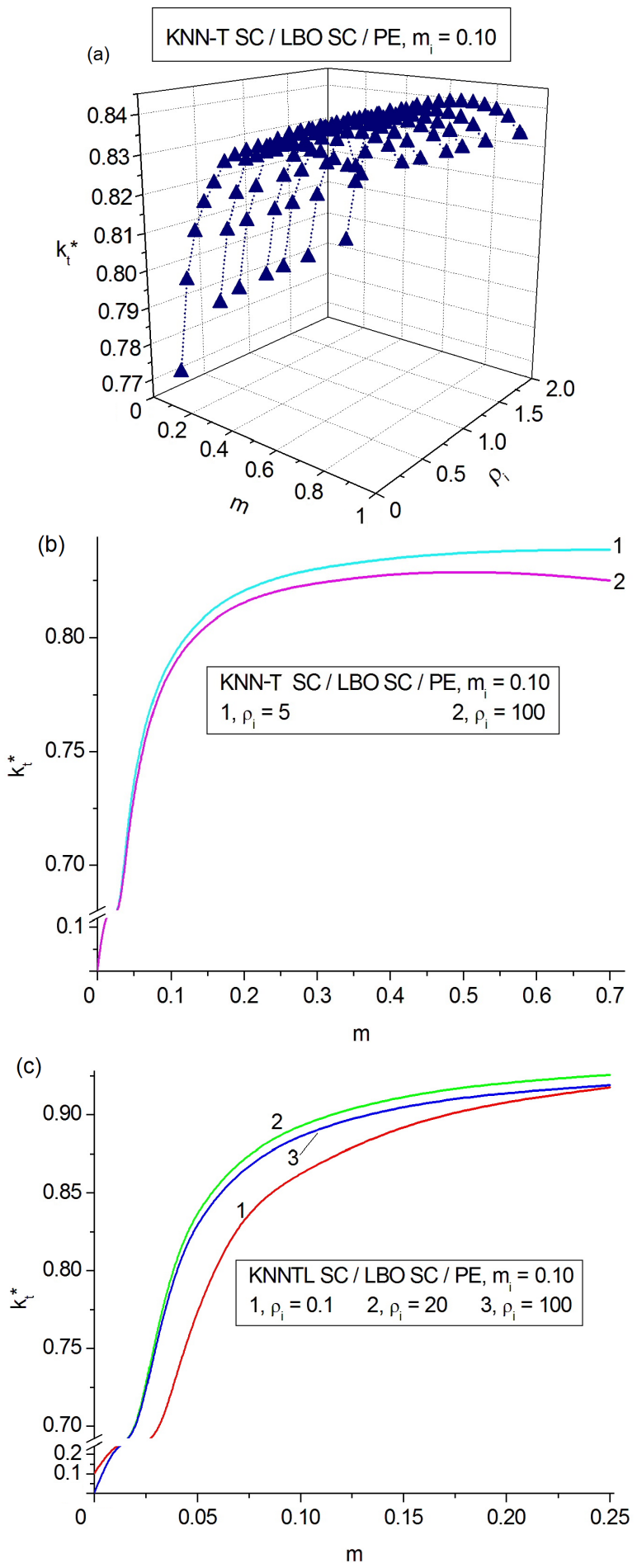

Fig. 6. ECF $k_{t}^{*}$ near local maximum (a and b) and in regions (c) where condition (7) holds

\section{E. Comparisons and Advantages}

We compare the effective parameters of the studied composites to the similar parameters of the related composites in two limiting cases. In the first limiting case, the 0-3 matrix is regarded as a piezo-passive medium because of assumption that the LBO SC inclusions are not piezoelectric. Results on the anisotropy factors $\zeta_{j}^{*}$ and ECF $k_{t}^{*}$ (Table IV) show that differences between the similar parameters are relatively small 
and decrease on increasing the volume fraction $m$. This is mainly caused by the high piezoelectric activity of the SC-1 rod in comparison to the surrounding $0-3$ matrix that exhibits either a weak piezoelectric effect or no piezoelectricity.

The second limiting case is concerned with $m_{i}=0$, i.e., with the 1-3 SC/ polymer composite with the piezo-passive matrix. Replacing the anisotropic $0-3 \mathrm{SC} /$ polymer matrix with the isotropic polymer matrix leads mainly to changes in the anisotropy factor $\zeta_{1}^{*}$ : for instance, no $\min \zeta_{1}^{*}(m)$ is observed on curve 1 in Fig. 7(a) and on curves 1 and 4 in Fig. 7(b). We remind the reader that $\min \zeta_{1}^{*}(m)$ in Fig. 2 (curves 1 and 4 ) and Fig. 4 (curves $1-5$ ) is related to the 1-0-3 composites wherein the 0-3 matrices are characterized by the definite elastic anisotropy. Values of $\min \zeta_{2}^{*}(m)$ and $\min \zeta_{3}^{*}(m)$ in Fig. 7(a) and (b) undergo minor changes in comparison to $\min \zeta_{2}^{*}(m)$ and $\min \zeta_{3}^{*}(m)$ in Fig. 2. These changes can be accounted for the larger volume fractions $m$ related to $\min \zeta_{2}^{*}(m)$ and $\min \zeta_{3}^{*}(m)$ in comparison to the small volume fractions $m$ related to $\min \zeta_{1}^{*}(m)$ in Fig. 2. The influence of the SC-1 rod with the high piezoelectric activity on the anisotropy factors from Eqs. (4) becomes stronger at larger $m$ values, while the piezo-passive polymer matrix in the 1-3 composite influences the transverse piezoelectric effect and, therefore, ECFs $k_{31}^{*}$ and $k_{p}^{*}$. Comparing the $k_{t}^{*}(m)$ dependences from Figs. 6 and 7(c), we state that these dependences undergo minor changes at replacing the matrix. The aforementioned changes of the elastic properties of the matrix do not lead to appreciable changes of $\max _{k_{t}^{*}}(m)$ that is achieved at large volume fractions of SC-1 $m>0.5$.

TABLE IV

ANISOTROPY FACTORS $\zeta_{j}^{*}$ AND ECF $k_{t}^{*}$ OF THE $1-0-3$ KNNTL SC / LBO $\mathrm{SC} / \mathrm{PE}$ COMPOSITE AT $m_{i}=$ CONST AND $\rho_{i}=$ CONST

\begin{tabular}{|c|c|c|c|c|c|c|}
\hline$\rho_{i}$ & $m_{i}$ & $m$ & $\zeta_{1}^{*}$ & $\zeta_{2}^{*}$ & $\zeta_{3}^{*}$ & $k_{t}^{*}$ \\
\hline \multirow[t]{10}{*}{10} & \multirow[t]{4}{*}{0.10} & 0.05 & $\begin{array}{c}-6.50 \\
(-6.98)\end{array}$ & $\begin{array}{c}-7.36 \\
(-7.39)\end{array}$ & $\begin{array}{c}-4.72 \\
(-4.74)\end{array}$ & $\begin{array}{c}0.841 \\
(0.841)\end{array}$ \\
\hline & & 0.10 & -6.15 & $\begin{array}{l}-7.68 \\
(-771)\end{array}$ & $\begin{array}{l}-4.98 \\
-500\end{array}$ & $\begin{array}{c}0.897 \\
0897\end{array}$ \\
\hline & & 0.25 & $\begin{array}{c}(-6.17) \\
-4.37\end{array}$ & $\begin{array}{c}(-7.71) \\
-6.79\end{array}$ & $\begin{array}{c}(-5.00) \\
-4.49\end{array}$ & $\begin{array}{c}(0.897) \\
0.927\end{array}$ \\
\hline & & & $(-4.38)$ & $(-6.80)$ & $(-4.49)$ & $(0.927)$ \\
\hline & \multirow[t]{6}{*}{0.20} & 0.05 & -8.31 & -7.27 & -4.66 & 0.825 \\
\hline & & & $(-8.39)$ & $(-7.33)$ & $(-4.71)$ & $(0.825)$ \\
\hline & & 0.10 & -7.10 & -7.26 & -4.71 & 0.889 \\
\hline & & & $(-7.14)$ & $(-7.30)$ & $(-4.73)$ & (0.888) \\
\hline & & 0.25 & -4.72 & -5.96 & -3.93 & 0.923 \\
\hline & & & $(-4.73)$ & $(-5.97)$ & $(-3.93)$ & $(0.923)$ \\
\hline \multirow[t]{11}{*}{100} & \multirow[t]{5}{*}{0.10} & 0.05 & -13.3 & -7.99 & $\begin{array}{l}5.37 \\
5.51\end{array}$ & 0.832 \\
\hline & & 0 & $\begin{array}{c}(-13.6) \\
-974\end{array}$ & $\begin{array}{c}(-8.19) \\
-6.96\end{array}$ & $\begin{array}{c}(-5.51) \\
-4.69\end{array}$ & $\begin{array}{c}(0.832) \\
0.889\end{array}$ \\
\hline & & & $(-9.86)$ & $(-7.05)$ & $(-4.75)$ & (0.889) \\
\hline & & 0.25 & -5.34 & -4.83 & -3.25 & 0.919 \\
\hline & & & $(-5.36)$ & $(-4.85)$ & $(-3.26)$ & (0.919) \\
\hline & \multirow[t]{6}{*}{0.20} & 0.05 & -15.0 & -6.81 & -4.59 & 0.812 \\
\hline & & & $(-15.6)$ & $(-7.06)$ & $(-4.76)$ & $(0.812)$ \\
\hline & & 0.10 & -10.3 & -5.55 & -3.73 & 0.875 \\
\hline & & & $(-10.5)$ & $(-5.64)$ & $(-3.79)$ & $(0.875)$ \\
\hline & & 0.25 & -5.38 & -3.72 & -2.47 & 0.907 \\
\hline & & & $(-5.41)$ & $(-3.74)$ & $(-2.48)$ & $(0.907)$ \\
\hline
\end{tabular}

${ }^{a}$ In the 4 th -7 th columns, values in parentheses are related to the composite with the piezo-passive LBO SC / PE matrix
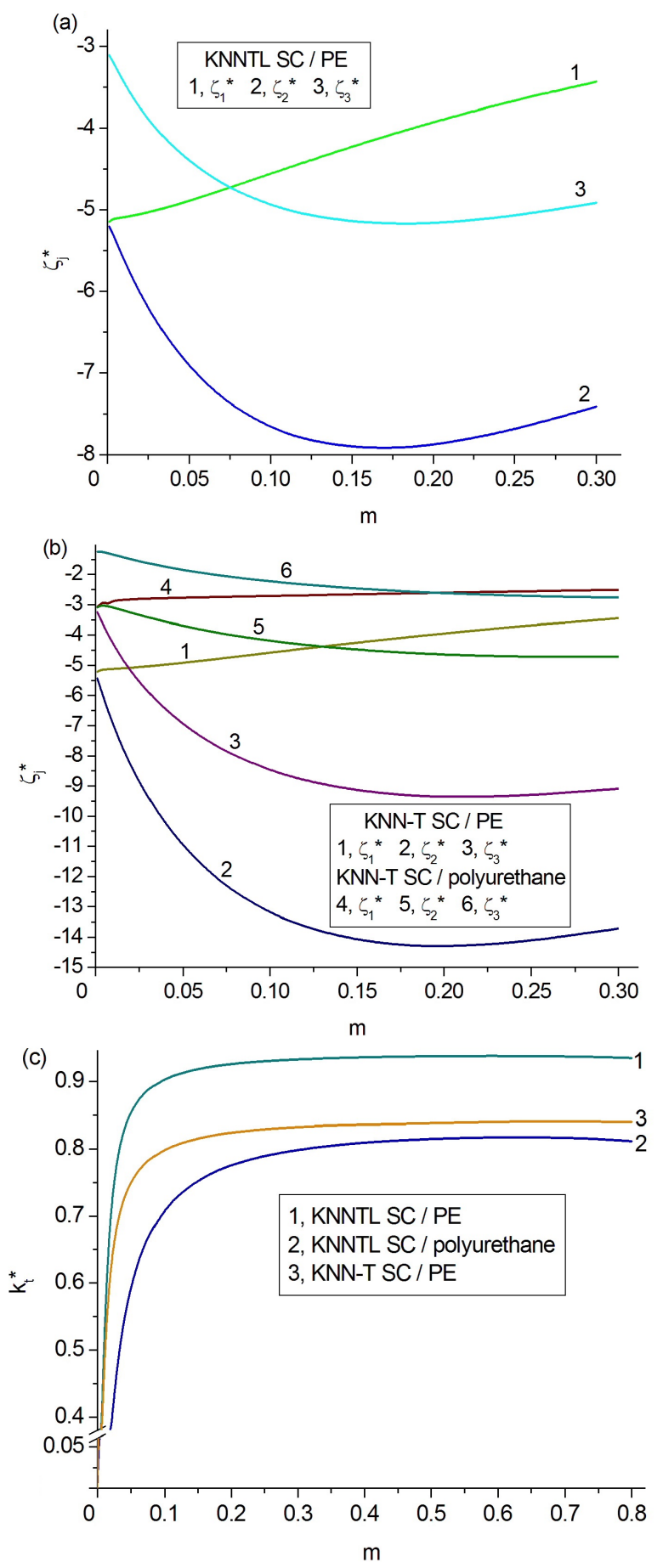

Fig. 7. Volume-fraction (m) dependence of anisotropy factors $\zeta_{j}^{*}$ and ECF $k_{t}^{*}$ of 1-3 SC / polymer composites

The large values of the studied parameters (see Figs. 2-4 and 6 , and Table IV) are achieved in the composites wherein the system of the heavily oblate LBO SC inclusions with $\rho_{i} \gg>1$ plays the important role. Such inclusions with specific sizes can be obtained, for example, at crystal growth by means of methods [28] and [29].

The studied PE-containing 1-0-3 composites with large $\left|\zeta_{j}^{*}\right|$ values have advantages over many piezo-active 1-3 composites. For example, a 1-3 lead-free niobate ceramic / 
epoxy composite is characterized by $\max _{k_{t}^{*}}^{*} \approx 0.5$ at $d_{33}^{*} \approx 200$ $\mathrm{pC} / \mathrm{N}$ [8]. For comparison, the 1-0-3 KNNTL SC / LBO SC / PE composite at $\rho_{i}=100$ and $m_{i}=0.10$ is characterized by $k_{t}^{*}=$ 0.832 and $d_{33}^{*}=265 \mathrm{pC} / \mathrm{N}(m=0.05), k_{t}^{*}=0.865$ and $d_{33}^{*}=310$ $\mathrm{pC} / \mathrm{N}(m=0.07)$, etc. We add that at the aforementioned $\rho_{i}, m_{i}$, and $m$ values, the simultaneous validity of conditions (5)-(7) is observed, see Fig. 2(a) and Table III. 1-3 lead-containing PMN-0.30PT SC / epoxy [30] and PMN-0.33PT SC / epoxy composites [31] were characterized by $\max k_{t}^{*} \approx 0.8$, and this experimental value is comparable to $\max k_{t}^{*}$ shown in Fig. 6(a) and (b). Finally, in a highly anisotropic modified $\mathrm{PbTiO}_{3}$ ceramic [12], the piezoelectric coefficient $d_{33}=56 \mathrm{pC} / \mathrm{N}$ is a few times smaller than $d_{33}^{*}$ of the studied 1-0-3 composites, and the anisotropy factor $\zeta_{1}$ of the modified $\mathrm{PbTiO}_{3}$ ceramic [27] is comparable to $\zeta_{1}^{*}$ of the composites.

\section{CONCLUSION}

We have provided the first analysis of the piezoelectric performance, electromechanical coupling, and anisotropy factors (4) of a 1-3-type lead-free SC-1 / SC-2 / polymer composite shown in Fig. 1. In the presence of two piezoelectric components, namely, domain-engineered ferroelectric SC-1 rods and piezoelectric SC-2 inclusions in a polymer matrix, the simultaneous fulfillment of conditions (5)-(7) for a large anisotropy of the piezoelectric properties and ECFs is observed; see, for instance, Fig. 2 and Table III. Since the maximum values of $\left|\zeta_{j}^{*}\right|$ are observed at relatively small volume fractions of the SC-1 $m$, as in Fig. 2, the role of the $0-3 \mathrm{SC}-2$ / polymer matrix in forming the anisotropic piezoelectric response and electromechanical coupling becomes highly important. This role is mainly concerned with the matrix elastic properties that can be varied in wide ranges when changing the aspect ratio $\rho_{i}$ and volume fraction $m_{i}$ of the SC-2 inclusions, see Fig. 5. It is shown that the larger $\left|\zeta_{j}^{*}\right|$ values are achieved in the case of the $0-3$ matrix that contains SC-2 and polymer components with a large difference of their elastic properties, i.e., the LBO SC and PE in the present study. The preferable aspect-ratio $\left(\rho_{i}\right)$ range at relatively small volume fractions $m_{i}$ in the $0-3$ matrix is $20 \leq \rho_{i} \leq 100$, and this is related to the presence of highly oblate SC-2 inclusions. In this $\rho_{i}$ range, the simultaneous validity of conditions (5)-(7) is observed at volume fractions of SC-1 $m$ (see Table III) that promote large ECFs $k_{33}^{*}$ and $k_{t}^{*}$.

The large $\left|\zeta_{j}^{*}\right|, k_{33}^{*}$, and $k_{t}^{*}$ values of the studied 1-3-type composites and their relatively large piezoelectric coefficient $d_{33}^{*} \sim 10^{2} \mathrm{pC} / \mathrm{N}$ are of significant interest for the selection of materials for piezoelectric transducers, acoustic antennas, and energy-harvesting devices with due respect to the environment.

\section{References}

[1] K. Ren, Y. Liu, X. Geng, H. F. Hofmann, and Q. M. Zhang, "Single crystal PMN-PT / epoxy 1-3 composite for energy-harvesting application“, IEEE Trans. Ultrason., Ferroelectr., a. Freq. Control, vol. 53, no. 3, pp. 631-638, Mar. 2006.

[2] S. Zhang, F. Li, J. Luo, R. Sahul, and T. R. Shrout, "Relaxor-PbTiO single crystals for various applications", IEEE Trans. Ultrason., Ferroelectr., a. Freq. Control, vol. 60, no. 8, pp.1572-1580, Aug. 2013.

[3] C. R. Bowen, V. Yu. Topolov, A. N. Isaeva, and P. Bisegna, "Advanced composites based on relaxor-ferroelectric single crystals: from electromechanical coupling to energy-harvesting applications", CrystEngComm, vol. 18, no. 32, pp. 5986-6001, Aug. 2016.

[4] V. Yu. Topolov, P. Bisegna, and C. R. Bowen, Piezo-Active Composites. Orientation Effects and Anisotropy Factors. Heidelberg, New York, Dordrecht, London: Springer, 2014.

[5] S. Gupta, D. Maurya, Y. Yan, and S. Priya. "Development of KNN-based piezoelectric materials". S. Priya and S. Nahm (eds.) Lead-Free Piezoelectrics. New York, Dordrecht, Heidelberg, London: Springer, 2012, pp. 89-119.

[6] A. Safari and M. Hejazi. "Lead-free KNN-based piezoelectric materials". S. Priya and S. Nahm (eds.) Lead-Free Piezoelectrics. New York, Dordrecht, Heidelberg, London: Springer, 2012, pp. 139-175.

[7] C.-H. Hong, H.-P. Kim, B.-Y. Ghoi, H.-S. Han, J.-S. Son, C.W. Ahn, and W. Jo, "Lead-free piezoceramics - Where to move on?", J. Materiomics, vol. 2, no. 1, pp. 1-24, Jan. 2016.

[8] Z.-Y. Shen, Y. Xu, and J.-F. Li, "Fabrication and electromechanical properties of microscale 1-3-type piezoelectric composites using $(\mathrm{Na}$, $\mathrm{K}) \mathrm{NbO}_{3}$-based Pb-free piezoceramics“, J. Appl. Phys., vol. 105, no. 10, p. 104103 - 4 p., May 2009.

[9] L.M. Zheng, X.Q. Huo, R. Wang, J.J. Wang, W.H. Jiang, W.W. Cao, "Large size lead-free $(\mathrm{Na}, \mathrm{K})(\mathrm{Nb}, \mathrm{Ta}) \mathrm{O}_{3}$ piezoelectric single crystal: growth and full tensor properties", CrystEngComm, vol. 15, no. 38, pp. 7718-7722, Jul. 2013.

[10] X. Huo, L. Zheng, R. Zhang, R. Wang, J. Wang, S. Sang, Y. Wang, B. Yang, and W. Cao, "A high quality lead-free $(\mathrm{Li}, \mathrm{Ta})$ modified $(\mathrm{K}$, $\mathrm{Na}) \mathrm{NbO}_{3}$ single crystal and its complete set of elastic, dielectric and piezoelectric coefficients with macroscopic $4 \mathrm{~mm}$ symmetry", CrystEng Comm, vol. 16, no. 42, pp. 9828-9833, Sep. 2014.

[11] X. Huo, R. Zhang, L. Zheng, S. Zhang, R. Wang, J. Wang, S. Sang, B. Yang, and W. Cao, "(K, Na, $\mathrm{Li})(\mathrm{Nb}, \mathrm{Ta}) \mathrm{O}_{3}: \mathrm{Mn}$ lead-free single crystal with high piezoelectric properties", J. Am. Cer. Soc., vol. 98, no. 6, pp. 1829-1835, Mar. 2015.

[12]Y. Xu, Ferroelectric Materials and Their Applications. Amsterdam: North-Holland, 1991.

[13] V. Yu. Topolov, C. R. Bowen, A. A. Panich, and A. N. Isaeva, "Piezoelectric sensitivity and hydrostatic response of novel lead-free 2-02 composites with two single-crystal components", Mater. Chem. Phys., vol. 201, no. 1, 224-234, Aug. 2017.

[14] V. Yu. Topolov and A. N. Isaeva, "High piezoelectric sensitivity and related parameters of a novel lead-free 1-0-3 composite", Ferroelectrics Lett. Sec., vol. 44, nos. 4-6, p. 73-80, Dec. 2017.

[15] V. Yu. Topolov, C. R. Bowen, A. N. Isaeva, and A. A. Panich, "Theoretical study on the piezoelectric performance of lead-free 1-3-type composites", Phys. Stat. Sol. A, vol. 215, no. 1, 1700548-11 p., Jan. 2018.

[16] V. Yu. Topolov, C. R. Bowen, and A. V. Krivoruchko, "Piezoelectric performance and hydrostatic parameters of novel 2-2-type composites", IEEE Trans. Ultrason., Ferroelectr., a. Freq. Control, vol. 64, no. 10, pp.1599-1607, Jun. 2017.

[17] T. Ikeda, Fundamentals of Piezoelectricity. Oxford: Oxford University Press, 1990.

[18] M. Adachi, T. Shiosaki, H. Kobayashi, O. Ohnishi, and A. Kawabata, "Temperature compensated piezoelectric lithium tetraborate crystal for high frequency surface acoustic wave and bulk wave device applications". Proceedings of 1985 IEEE Ultrasonics Symposium. New York: IEEE, 1985, pp. 228-232.

[19] K. E. Evans and K. L. Alderson, "The static and dynamic moduli of auxetic microporous polyethylene“, J. Mater. Sci. Lett., vol. 11, no. 24, pp. 1721-1724, Dec. 1992.

[20] I. N. Groznov, "Dielectric permittivity". A. M. Prokhorov (ed.-i.-chief) Physics Encyclopaedia. Moscow: Sovetskaya Entsiklopediya, 1983, pp. 178-179 (in Russian).

[21] L. V. Gibiansky and S. Torquato, "On the use of homogenization theory to design optimal piezocomposites for hydrophone applications", $J$. Mech. Phys. Solids, vol. 45, no. 5, pp. 689-708, May 1997. 
[22] K. Uchino, Piezoelectric Actuators and Ultrasonic Motors. Boston, Dordrecht, London: Kluwer Academic Publishers, 1996.

[23] S.-H. Wang and M.-C. Tsai, "Dynamic modeling of thickness-mode piezoelectric transducer using the block diagram approach", Ultrasonics, vol. 51, no. 5, pp. 617-624, Jul. 2001.

[24] Y. Roh and C. Moon, "Design and fabrication of an ultrasonic speaker with thickness mode piezoceramic transducers", Sens. a. Actuat. A, vol. 99, no. 3, pp. 321- 326, Jun. 2002.

[25] H. L. W. Chan and J. Unsworth, "Simple model for piezoelectric ceramic / polymer 1-3 composites used in ultrasonic transducer applications". IEEE Trans. Ultrason., Ferroelectr., a. Freq. Control, vol. 36, no. 4, pp. 434-441, Apr. 1989.

[26] V. G. Gavrilyachenko and E. G. Fesenko, "Piezoelectric effect of lead titanate single crystals", Kristallografiya, vol. 16, no. 3, pp. 640-641, Jun. 1971 (in Russian); Soviet Phys. - Crystallogr., vol. 16, no. 3, pp. 549 $550,1971$.

[27] A. V. Turik and V. Yu. Topolov, "Ferroelectric ceramics with a large piezoelectric anisotropy“, J. Phys. D: Appl. Phys., vol. 30, no. 11, pp. 1541-1549, Jun. 1997.

[28] N. Tsutsui, Y. Ino, K. Imai, N. Senguttuvan, and M. Ishii, "Growth of high quality 4 in diameter $\mathrm{Li}_{2} \mathrm{~B}_{4} \mathrm{O}_{7}$ single crystals", J. Cryst. Growth, vol. 229, nos. 1-4, pp. 283-288, Jul. 2001.

[29] J. Xu, S. Fan, and B. Lu, "Growth of $\varnothing 4$ " $\mathrm{Li}_{2} \mathrm{~B}_{4} \mathrm{O}_{7}$ single crystals by multi-crucible Bridgman method“, J. Cryst. Growth, vol. 264, nos. 1-3, pp. 260-265, Mar. 2004.

[30] F. Wang, C. He, Y. Tang, X. Zhao, and H. Luo, "Single-crystal $0.7 \mathrm{~Pb}\left(\mathrm{Mg}_{1 / 3} \mathrm{Nb}_{2 / 3}\right) \mathrm{O}_{3}-0.3 \mathrm{PbTiO}_{3} /$ epoxy 1-3 piezoelectric composites prepared by the lamination technique“, Mater. Chem. Phys., vol. 105, no. 1, pp. 273-277, Jan. 2007.

[31] K. C. Cheng, H. L. W. Chan, C. L. Choy, Q. Yin, H. Luo, and Z. Yin, "Single crystal PMN-0.33PT / epoxy 1-3 composites for ultrasonic transducer applications", IEEE Trans. Ultrason., Ferroelectr., a. Freq. Control, vol. 50, no. 9, pp. 1177-1183, Sep. 2003.

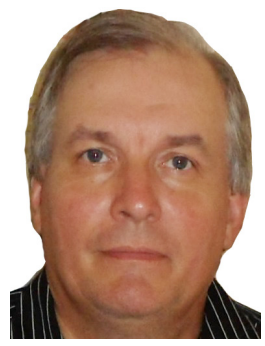

Vitaly Yu. Topolov was born in Rostov-on-Don, Russia, in 1961. He received the qualification "Physicist. Educator" (Hons., 1984) and degrees "Candidate of Sciences (Physics and Mathematics)" (1987) and "Doctor of Sciences (Physics and Mathematics)" (2000), all from the Rostov State University, Russia. Since 2006, he has been a Professor with the Department of Physics, Southern Federal University, Rostov-on-Don. He has authored five monographs, three edited conference proceedings, and about

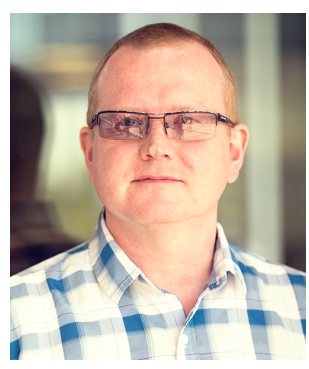
420 papers, chapters in monographs, reviews, conference proceedings, and abstracts. His current research interests include heterogeneous ferroelectrics, smart materials, domain and heterophase structures, and electromechanical effects in piezoactive composites.

Christopher R. Bowen was born in Beddau, South Wales, UK, in 1968. He received the B. Sc. (First Class) degree in materials science from the School of Materials, University of Bath, Bath, UK, in 1990, and the Ph. D. degree from the Department of Materials, University of Oxford, Oxford, UK. He joined the University of Bath, in 1998, where he is currently a Professor. He has authored three monographs, over 260 papers, chapters in monographs,

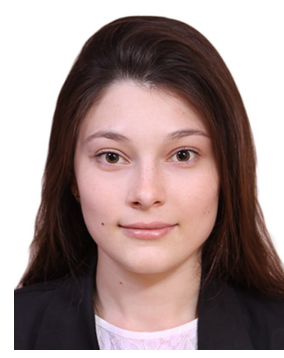
conference proceedings, and abstracts. His current research interests include the manufacture and characterization of ferroelectric ceramics and piezo-active composites for sensor, actuator, and energy-harvesting applications.

Ashura N. Isaeva was born in Rostov-on-Don, Russia, in 1995. She received the B. Sc. degree and qualification "Technical Physics" from the Department of Physics, Southern Federal University, Rostov-on-Don, Russia, in 2016. Since September 2016, she is a master-course student at the International Research Center "Smart Materials", Southern Federal University, Rostov-on-Don, Russia. She has authored 10 papers, conference proceedings, and abstracts. Her current research interests include piezo-active composites for transducer, hydroacoustic, energy-harvesting, and other applications. 